\title{
How Do Uncertainties in the Surface Chemical Composition of the Sun Affect the Predicted Solar Neutrino Fluxes?
}

\author{
John N. Bahcall and Aldo M. Serenelli \\ Institute for Advanced Study, Einstein Drive, Princeton, NJ 08540
}

\begin{abstract}
We show that uncertainties in the values of the surface heavy element abundances of the Sun are the largest source of the theoretical uncertainty in calculating the p-p, pep, ${ }^{8} \mathrm{~B},{ }^{13} \mathrm{~N},{ }^{15} \mathrm{O}$, and ${ }^{17} \mathrm{~F}$ solar neutrino fluxes. Our results focus attention on the necessity for improving the measurement of heavy element abundances while at the same time reducing the estimated uncertainties in the predicted solar neutrino fluxes due to abundance errors. We evaluate for the first time the sensitivity (partial derivative) of each solar neutrino flux with respect to the surface abundance of each element. We then calculate the uncertainties in each neutrino flux using the preferred 'conservative' (based upon changes of measured values with time) and 'optimistic' (current values) estimates for the uncertainties in the element abundances. The total conservative (optimistic) composition uncertainty in the predicted ${ }^{8} \mathrm{~B}$ neutrino flux is $11.6 \%$ (5.0\%) when sensitivities to individual element abundances are used. The traditional method that lumps all abundances into a single quantity (total heavy element to hydrogen ratio, $Z / X)$ yields a larger uncertainty, 20\%. The uncertainties in the carbon, oxygen, neon, silicon, sulphur, and iron abundances all make significant contributions to the uncertainties in calculating solar neutrino fluxes; the uncertainties of different elements are most important for different neutrino fluxes. The uncertainty in the iron abundance is the largest source of the estimated composition uncertainties of the important ${ }^{7} \mathrm{Be}$ and ${ }^{8} \mathrm{~B}$ solar neutrinos. Carbon is the largest contributor to the uncertainty in the calculation of the p-p, ${ }^{13} \mathrm{~N}$, and ${ }^{15} \mathrm{O}$ neutrino fluxes. However, for all neutrino fluxes, several elements contribute comparable amounts to the total composition uncertainty.
\end{abstract}

\section{INTRODUCTION}

What is the role of the chemical composition of the surface of the Sun in the calculation of the solar neutrino fluxes? 
In 1966, almost four decades ago, the role of the chemical composition was described as follows: "The primordial (or surface) composition assumed in computing the solar models represents the largest recognized uncertainty in the predicted capture rate;..." (Bahcall 1966). Recently, in 2004, the role of the chemical composition was summarized as follows: "The surface chemical composition of the Sun is the most problematic and important source of uncertainties." (Bahcall \& Pinsonneault 2004).

Some things change very slowly.

\subsection{Previous calculations using total heavy element abundance $Z$}

From the very beginning of solar neutrino calculations, the chemical composition of the Sun has been a principal source of uncertainty (see Sears 1964). The first systematic investigations of the effect of composition uncertainties on the important ${ }^{8} \mathrm{~B}$ solar neutrino flux concluded that the calculated neutrino flux was uncertain by a factor of two due to uncertainties in the chemical composition (Bahcall et al. 1967; Bahcall et al. 1968).

In the early days of solar neutrino calculations, the uncertainty in the neutrino fluxes due to the chemical composition was evaluated by calculating the sensitivity of the different fluxes to different choices of the total heavy element abundance (Sears 1964; Bahcall 1964; Bahcall et al. 1967; Bahcall et al. 1968; Abraham \& Iben 1971). More refined calculations were eventually carried out using the logarithmic partial derivatives of the neutrino fluxes, $\phi_{i}$, with respect to the total heavy element abundance by mass, $Z$, i.e., $\partial \ln \phi_{i} / \partial \ln Z$ (Bahcall \& Ulrich 1971).

The abundances of heavy elements in the solar photosphere are determined as ratios, the ratio of an individual heavy element abundance to the abundance of hydrogen, $X$. The abundances are linked by the relation $X+Y+Z=1$, where $Y$ is the surface abundance of helium. The fact that the abundances are measured as ratios was first taken into account in the paper by Bahcall et al. (1982), who calculated partial derivatives $\partial \ln \phi_{i} / \partial \ln (Z / X)$. In the intervening two decades, there have been many evaluations of the uncertainty of the solar neutrino fluxes caused by the solar composition. However, these evaluations all used variations with respect to the total heavy element abundance, $Z$, or with respect to $Z / X$, the total heavy element abundance divided by the hydrogen abundance.

There has not been a previous systematic investigation of the uncertainty of solar neutrino fluxes due to individual heavy element abundances. All of the recent papers with which we are familiar estimate the uncertainty due to the solar composition by considering variations in the total heavy element abundance, $Z$, or in $Z / X$ (see, e.g., Schlattl \& Weiss 
1999; Bahcall et al. 2001; Watanabe \& Shibahashi 2001; Fiorentini \& Ricci 2002; Couvidat et al. 2003; Boothroyd \& Sackmann 2003; Bahcall \& Pinsonneault 2004; Young \& Arnett 2005).

Some things change very slowly.

\subsection{Necessity of evaluating uncertainties due to individual elements}

It has long been recognized that changes in the abundances of some heavy elements, e.g., iron or silicon, affect the calculated solar neutrino fluxes more than do the abundances of other elements (see discussion in § IV.D of Bahcall et al. 1982). This is largely because the heavier elements are highly ionized only in the solar core, where they affect directly the calculated radiative opacity and indirectly the solar neutrino fluxes. The lighter, volatile elements affect the radiative opacity most dramatically in the region somewhat below the convective zone (temperatures somewhat above $2 \times 10^{6} \mathrm{~K}$ ). Oxygen is a principal contributor to the radiative opacity just below the convective zone (Turcotte \& Christensen-Dalsgaard 1998).

In recent years, determinations of the solar abundances of heavy elements have become more refined and detailed (Grevesse \& Sauval 1998, 2000; Lodders 2003) and especially (Asplund et al. 2000; Asplund 2000; Allende Prieto et al. 2001, 2002; Asplund et al. 2004; Asplund et al. 2005). These recent determinations yield significantly lower values than were previously adopted (e.g., by Grevesse \& Sauval 1998) for the abundances of the volatile heavy elements: C, N, O, Ne, and Ar. However, these recent abundance determinations lead to solar models that disagree with helioseismological measurements (Bahcall \& Pinsonneault 2004; Basu \& Antia 2004). For example, the calculated depth of the convective zone differs by about $15 \sigma$ from the measured value and the calculated surface helium abundance differs from the measured value by about $7 \sigma$ (see equations 1 and 2 of Bahcall, Serenelli \& Basu 2005). Detailed and refined recalculations of the radiative opacity by the Opacity Project collaboration disfavor (Seaton \& Badnell 2004; Badnell et al. 2004) the suggestion (Basu \& Antia 2004; Bahcall et al. 2004b; Bahcall et al. 2005) that the origin of the discrepancy might be the adopted opacities rather than the adopted heavy element abundances.

The discrepancies between helioseismological measurements and the predictions made using recent determinations of heavy elements make it especially important that the effect of individual element uncertainties be evaluated. The discrepancies occur in the temperature region below the solar convective zone, $2 \times 10^{6} \mathrm{~K}$ to $4.5 \times 10^{6} \mathrm{~K}$ (Bahcall et al. 2005). In this temperature domain, the volatile heavy elements, $\mathrm{C}, \mathrm{N}, \mathrm{O}, \mathrm{Ne}$, and $\mathrm{Ar}$, are partially 
ionized and their abundances significantly affect the radiative opacities. We need to separate out the effects of the volatile element abundances that contribute to the helioseismological discrepancies from the effects of abundances, e.g., Si and Fe, that are most important in the solar core.

\subsection{What do we do in this paper?}

We derive for the first time in this paper individual uncertainties in each neutrino flux due to each of the important heavy elements in the solar composition. We then estimate 'conservative' uncertainties by assuming that the differences between the most recent abundance determinations and the previous abundance determination represent $1 \sigma$ uncertainties. We also use more 'optimistic' uncertainties taken from the most recent review of abundance determinations (Asplund et al. 2005).

We then combine the effects of all composition uncertainties to determine the net effect of composition uncertainties on each solar neutrino flux and on the rate of each radiochemical solar neutrino experiment. Finally, we combine the effects of all known sources of uncertainties, including composition uncertainties, on each neutrino flux and experimental radiochemical rate. We identify the heavy elements that most strongly affect the predicted neutrino fluxes and we identify for which solar neutrino fluxes composition uncertainties are most important.

Abundance determinations for the Sun change frequently as improved techniques, new atomic data, and more observations become available. Therefore, we describe in this paper the steps necessary to make detailed and reliable estimates of the uncertainties in the solar neutrino fluxes for a given set of abundances and their uncertainties. We also make available at http://www.sns.ias.edu/ jnb computer code and numerical data that can facilitate future investigations when new abundance determinations are published.

We discuss the role of correlations between the uncertainties of different elements. This is an aspect of abundance discussions that is not treated explicitly in any of the papers with which we are familiar. However, we will show that for future precision evaluations of the effects of abundance determinations on neutrino fluxes we must know the correlations, if any, between the quoted abundance determinations and their uncertainties.

We use for the calculations in this paper the recently computed standard solar models BP04 and BP04+ (Bahcall \& Pinsonneault 2004), which are described below. However, we also verify that small changes such as occur between different recent redeterminations of the solar abundances cause only negligible changes (typically $0.1 \%$ ) in the estimated 
uncertainties of the solar neutrino fluxes. Table 1 of Bahcall et al. (2004b) lists the specific element abundances adopted in computing each of the solar models BP04 and BP04+.

The plan of the paper is as follows. In $\S 2$, we present and discuss the logarithmic partial derivatives of each solar neutrino flux with respect to each of the ratios (major heavy element abundance)/(hydrogen abundance). The results are given for two separate solar models, BP04 (which uses the Grevesse \& Sauval 1988 composition) and BP04+ (which uses more recent determinations of the abundances of the volatile elements). We also present the partial derivatives with respect to the heavy element to hydrogen ratio, $Z / X$. The robustness of the partial derivatives with respect to $Z / X$ is made evident from the very small change in their numerical values over more than two decades, although the solar models have been greatly refined.

We present in $\S 3$ 'conservative' and 'optimistic' estimates for the current uncertainties in the heavy element abundances. We adopt as our preferred choice the conservative uncertainty estimates. We compute in $\S 4$ the uncertainties in individual neutrino fluxes due to the uncertainties in each heavy element abundance. We combine for each neutrino flux in $\S 5$ the uncertainties from all abundance uncertainties; these results are summarized in Table 7. Our bottom line is given in Table 8 of $\S 6$, where we present the total uncertainties in the neutrino fluxes for different methods of calculation.

We summarize and discuss our main results in $\S 7$.

We recommend that all readers start by perusing $\S 7$, our summary and discussion section. For many readers, $\S 7$ contains all they need to know about the subject.

\section{LOGARITHMIC DERIVATIVES}

We define in $\S 2.1$ the logarithmic partial derivatives of each of the neutrino fluxes with respect to each of the element abundances. We present in $\S 2.2$ newly calculated partial derivatives that were obtained using the recently-derived BP04 and BP04+ solar models (Bahcall \& Pinsonneault 2004). We also calculate in $\S 2.3$ new values for the logarithmic partial derivatives with respect to the total heavy element to hydrogen ratio, $Z / X$. We compare these newly-calculated values with partial derivatives that were obtained using 1982 and 1988 solar models. Finally, we use the results of the previous subsection to explain, in $\S 2.4$, the reason why estimates of the composition uncertainties based upon historical changes in $Z / X$ have led to overestimates of the composition uncertainties in the calculated solar neutrino fluxes. 
Table 1: Partial derivatives of neutrino fluxes with respect to composition fractions. The entries in the table are the logarithmic partial derivatives, $\alpha_{i j}$ of the solar neutrino fluxes, $\phi_{i}$, with respect to the fractional abundances of the heavy elements, $\beta_{j}$ (see eq. [1] and eq. [2]). The partial derivatives were computed using the solar model BP04 (Bahcall \& Pinsonneault 2004). The derivatives given here are available in digital form at http://www.sns.ias.edu/ jnb under the menu items Solar Neutrinos/software and data.

\begin{tabular}{lccccccccc}
\hline \hline Source & $\mathrm{C}$ & $\mathrm{N}$ & $\mathrm{O}$ & $\mathrm{Ne}$ & $\mathrm{Mg}$ & $\mathrm{Si}$ & $\mathrm{S}$ & $\mathrm{Ar}$ & $\mathrm{Fe}$ \\
\hline $\mathrm{pp}$ & -0.014 & -0.003 & -0.006 & -0.005 & -0.005 & -0.011 & -0.008 & -0.002 & -0.023 \\
pep & -0.025 & -0.006 & -0.011 & -0.005 & -0.005 & -0.014 & -0.017 & -0.006 & -0.065 \\
hep & -0.015 & -0.004 & -0.023 & -0.017 & -0.018 & -0.037 & -0.028 & -0.007 & -0.069 \\
${ }^{7} \mathrm{Be}$ & -0.002 & 0.002 & 0.052 & 0.049 & 0.051 & 0.104 & 0.074 & 0.018 & 0.209 \\
${ }^{8} \mathrm{~B}$ & 0.030 & 0.011 & 0.121 & 0.096 & 0.096 & 0.194 & 0.137 & 0.034 & 0.515 \\
${ }^{13} \mathrm{~N}$ & 0.845 & 0.181 & 0.079 & 0.057 & 0.060 & 0.128 & 0.094 & 0.024 & 0.342 \\
${ }^{15} \mathrm{O}$ & 0.826 & 0.209 & 0.093 & 0.068 & 0.070 & 0.150 & 0.109 & 0.028 & 0.401 \\
${ }^{17} \mathrm{~F}$ & 0.033 & 0.010 & 1.102 & 0.076 & 0.078 & 0.164 & 0.120 & 0.031 & 0.444 \\
\hline
\end{tabular}

\subsection{Definition of partial derivatives}

The sensitivity of the neutrino fluxes, $\phi_{i}$, to the input parameters, $\beta_{j}$, can be expressed to high accuracy in terms of the logarithmic partial derivatives, $\alpha_{i j}$ (see Bahcall \& Ulrich 1988; Bahcall 1989). The logarithmic derivatives are defined by the equation

$$
\alpha_{i j}=\frac{\partial \ln \phi_{i}}{\partial \ln \beta_{j}}
$$

In this paper, we are primarily concerned with the uncertainties in the calculated solar neutrino fluxes that result from uncertainties in the solar heavy element abundances. Thus we concentrate on partial derivatives in which the $\beta_{j}$ are the mass fractions of different heavy elements relative to the hydrogen mass fraction. Thus,

$$
\beta_{j}=\frac{\text { (mass fraction of element } \mathrm{j} \text { ) }}{\text { (mass fraction of hydrogen) }} .
$$

It is conventional to denote the mass fraction of hydrogen by $X$.

We note that the partial derivatives give rise to the power law dependences of neutrino fluxes upon model parameters that are widely used in the literature. We have

$$
\phi_{i}=\phi_{i}(0)\left[\frac{\beta_{j}}{\beta_{j}(0)}\right]^{\alpha_{i j}} .
$$


In practice, the partial derivatives are computed by first evolving a standard solar model with a specific set of input data. The standard solar model is used to predict the best-estimate set of neutrino fluxes, $\phi_{i}(0)$. Then at least one additional solar model is evolved in which one parameter, $\beta_{i}$, is changed from its standard value. If only these two solar models are available, then the logarithmic partial derivative $\alpha_{i j}$ can be estimated from the following equation:

$$
\alpha_{i j} \simeq \frac{\ln \left[\phi_{i} / \phi_{i}(0)\right]}{\ln \left[\beta_{j} / \beta_{j}(0)\right]} .
$$

For this paper, we have used five solar models, including the standard model, in evaluating each of the derivatives $\alpha_{i j}$. Except for the case of argon, we have compared solar models in which the abundance fractions differed from the standard abundance fractions by -0.10 dex, -0.05 dex, 0.0 dex, +0.05 dex, and +0.10 dex. Argon is much less abundant than the other elements we consider; for this case only, we compared models with abundance fractions that differed by -0.20 dex, -0.10 dex, 0.0 dex, +0.10 dex, and +0.20 dex from the standard abundance.

For each modified composition, i.e. for each +-0.05, +-0.10 change in each of the elements (and +-0.10, +-0.20 for argon), we have calibrated the corresponding solar model to the present solar radius and luminosity and to the corresponding $\mathrm{Z} / \mathrm{X}$ value (the latter value being different for each modified composition). Opacity tables were recalculated for each different mixture.

\subsection{The calculated individual partial derivatives}

Table 1 presents the partial derivatives computed using the standard solar model BP04 (Bahcall \& Pinsonneault 2004) that assumes the Grevesse \& Sauval (1998) solar abundances. Table 2 presents the partial derivatives computed using the solar model BP04+ (Bahcall \& Pinsonneault 2004) that uses recent determinations for the volatile elements $\mathrm{C}, \mathrm{N}, \mathrm{O}, \mathrm{Ne}$, and Ar rather than the Grevesse \& Sauval (1998) values for these abundances.

The derivatives of the important ${ }^{7} \mathrm{Be},{ }^{8} \mathrm{~B}$, and pp solar neutrino fluxes with respect to heavy elements like iron are much larger than the derivatives with respect to the volatile elements like $\mathrm{C}, \mathrm{N}$, and $\mathrm{O}$. The heavier elements are ionized at the higher temperatures characteristic of the solar interior, where the neutrinos are formed, while the volatile elements can be ionized at the lower temperatures that are characteristic of the region below the solar convective zone. Thus, for a given fractional uncertainty, the iron, silicon, and sulphur abundances more strongly affect the calculated neutrino fluxes while the C, N, and O abundances more strongly affect the comparison with helioseismology. 
Table 2: Same as Table 1 but for a solar model with BP04+ (Bahcall \& Pinsonneault 2004) composition. The volatile elements C, N, O, Ne, and Ar in this model have the lower abundances determined recently (Asplund et al. 2000; Asplund 2000; Allende Prieto et al. 2001, 2002; Asplund et al. 2004) rather than the previously standard Grevesse \& Sauval (1998) abundances assumed in computing Table 1.The derivatives given here are available in digital form at http://www.sns.ias.edu/ jnb under the menu items Solar Neutrinos/software and data.

\begin{tabular}{lccccccccc}
\hline \hline Source & $\mathrm{C}$ & $\mathrm{N}$ & $\mathrm{O}$ & $\mathrm{Ne}$ & $\mathrm{Mg}$ & $\mathrm{Si}$ & $\mathrm{S}$ & $\mathrm{Ar}$ & $\mathrm{Fe}$ \\
\hline $\mathrm{pp}$ & -0.010 & -0.003 & -0.005 & -0.003 & -0.005 & -0.010 & -0.007 & -0.001 & -0.022 \\
pep & -0.018 & -0.004 & -0.008 & -0.002 & -0.003 & -0.015 & -0.015 & -0.003 & -0.062 \\
hep & -0.012 & -0.003 & -0.018 & -0.011 & -0.019 & -0.039 & -0.029 & -0.005 & -0.072 \\
${ }^{7} \mathrm{Be}$ & 0.005 & 0.002 & 0.046 & 0.033 & 0.057 & 0.115 & 0.080 & 0.012 & 0.230 \\
${ }^{8} \mathrm{~B}$ & 0.035 & 0.009 & 0.099 & 0.064 & 0.107 & 0.212 & 0.150 & 0.023 & 0.553 \\
${ }^{13} \mathrm{~N}$ & 0.846 & 0.180 & 0.055 & 0.036 & 0.065 & 0.139 & 0.102 & 0.015 & 0.355 \\
${ }^{15} \mathrm{O}$ & 0.824 & 0.211 & 0.068 & 0.043 & 0.077 & 0.163 & 0.119 & 0.018 & 0.423 \\
${ }^{17} \mathrm{~F}$ & 0.035 & 0.008 & 1.074 & 0.048 & 0.086 & 0.180 & 0.130 & 0.020 & 0.469 \\
\hline
\end{tabular}

\subsection{Partial derivatives with respect to total $Z / X$}

Table 3 presents the partial derivatives with respect to the total heavy element to hydrogen ratio, $Z / X$. The $Z / X$ derivatives are given here for completeness and for comparison with previous results. The partial derivatives in Table 3 are defined by the relation

$$
\alpha_{i}(Z / X)=\frac{\partial \ln \phi_{i}}{\partial \ln (Z / X)}
$$

When computing the partial derivatives in equation (5), we change $Z / X$ by multiplying all elements by the same factor, i.e., we increase (or decrease) the abundance of all heavy elements by the same fractional amount.

We present values for the partial derivatives that are computed not only with the recent BP04 and BP04+ solar models (columns [2] and [3] of Table 3, respectively), but also the values that were computed and published using the 1988 standard solar model (Bahcall \& Ulrich 1988, column [4]) and the 1982 standard solar model (Bahcall et al. 1982, column [5]). We note that the 1982 and 1988 models did not include element diffusion, used older (Los Alamos) opacities and nuclear reaction rates.

The remarkable robustness of the logarithmic partial derivatives can be seen by eye by comparing the different columns of Table 3. The rms fractional difference between the partial 
Table 3: $Z / X$ : Partial derivatives of neutrino fluxes with respect to the total heavy element to hydrogen ratio. The entries in the table are the logarithmic partial derivatives, $\alpha_{i}(Z / X)$ of the solar neutrino fluxes, $\phi_{i}$, with respect to the the heavy element to hydrogen ratio $Z / X$ (see eq. [5]). The partial derivatives were computed using the BP04 solar model (column [2]), the BP04+ solar model (column [3]), the Bahcall \& Ulrich (1988) standard model (Table XV of BU88, column [4]), and the Bahcall et al. (1982) standard model (Table XI of Bahcall et al. 1982, column [5]).

\begin{tabular}{lrrrc}
\hline \hline Source & BP04 & BP04+ & BU88 & Bahcall et al. 82 \\
\hline pp & -0.084 & -0.071 & -0.08 & -0.05 \\
pep & -0.171 & -0.147 & -0.17 & \\
hep & -0.242 & -0.229 & -0.22 & \\
${ }^{7} \mathrm{Be}$ & 0.619 & 0.637 & 0.58 & 0.60 \\
${ }^{8} \mathrm{~B}$ & 1.364 & 1.369 & 1.27 & 1.26 \\
${ }^{13} \mathrm{~N}$ & 1.897 & 1.870 & 1.86 & 1.67 \\
${ }^{15} \mathrm{O}$ & 2.056 & 2.038 & 2.03 & 2.00 \\
${ }^{17} \mathrm{~F}$ & 2.169 & 2.151 & 2.09 & \\
\hline
\end{tabular}

derivatives $\partial \ln \phi_{i} / \partial \ln (Z / X)$ computed by Bahcall \& Ulrich (1988) and the values computed using the BP04 solar model is $1.9 \%$. Even if we go all the way back to the first computation, by Bahcall et al. (1982), the rms fractional difference between the 1982 and the 2004 values (columns [4] and [2], respectively) is only 10.6\%. The robustness of the partial derivatives is due in part to the fact that all of the models are calibrated to the relatively accurately known solar luminosity and age.

Table 3 implies that we would have made only of order a $10 \%$ error in the estimated total flux uncertainties due to composition uncertainties had we used the 1982 partial derivatives instead of their 2004 values. In all cases, using the original 1982 partial derivatives would have changed by less than $1 \%$ the total flux uncertainties we estimate due to composition uncertainties.

We conclude that the major revisions and refinements that have been implemented in standard solar models over the past two and a half decades have not significantly affected $\partial \ln \phi_{i} / \partial \ln (Z / X)$. 


\subsection{Why $Z / X$ changes give an overestimate of composition changes}

The abundances of the heavier elements like $\mathrm{Si}, \mathrm{S}$, and $\mathrm{Fe}$ are relatively well known and therefore these elements do not affect greatly $\Delta(Z / X) /(Z / X)$, although they do affect strongly the calculated neutrino fluxes. The influence of the heavy elements like silicon, sulphur, magnesium, and iron on the calculated neutrino fluxes is primarily through their affect on the radiative opacity in the core of the solar model. As we shall see in the following section (see especially Table 4), the abundances of the light elements like C, N, O, and $\mathrm{Ne}$ are relatively poorly known and therefore they contribute a large amount to the fractional uncertainty, $\Delta(Z / X) /(Z / X)$. However, these light elements do not affect the solar neutrino fluxes very much (Bahcall \& Pinsonneault 2004); they are ionized near the base of the convective zone, outside the region where the neutrinos are formed.

Thus some elements, like $\mathrm{C}$ and $\mathrm{O}$, affect strongly $\Delta(Z / X) /(Z / X)$ without affecting very much the calculated neutrino fluxes. Conversely, heavier elements like Fe, affect the neutrino fluxes very significantly but, because their abundances are relatively well known, not $\Delta(Z / X) /(Z / X)$.

The imperfect correlation between changes in $\Delta(Z / X) /(Z / X)$ and changes in calculated

neutrino fluxes is the basic reason that it is necessary to evaluate individually the effects of individual abundance uncertainties on the flux estimates.

We shall compute and present in what follows the flux uncertainties calculated using the partial derivatives from Table 1, Table 2, and Table 3. We will adopt the results obtained with Table 1 for our preferred estimates of uncertainties since the BP04 solar model is in good agreement with helioseismology while the $\mathrm{BP} 04+$ solar model is in poor agreement with helioseismological measurements in the region between a solar radius of $R=0.7 R_{\odot}$ and $R=0.4 R_{\odot}$ (Bahcal et al. 2005). We also prefer the estimated uncertainties obtained with the derivatives of Table 1 because these uncertainties are more conservative (larger) than the estimates obtained with Table 2.

\section{ABUNDANCE UNCERTAINTIES}

In this section, we discuss and present two different estimates for the uncertainties in the heavy element abundances on the surface of the Sun. Our 'conservative' uncertainty estimates are obtained by comparing recent abundance analyses with previously standard analyses. Our more 'optimistic' uncertainty estimates are obtained by adopting the uncertainty estimates in the most recent review (Asplund et al. 2005). 


\subsection{Element abundances}

Recent analyses of the surface chemical composition of the Sun use three-dimensional atmospheric models, take account of hydrodynamic effects, and pay special attention to uncertainties in the atomic data and the observed spectra. Mass fractions that are lower than the previous standard values (Grevesse \& Sauval 1998) have been obtained in this way for C, N, O, Ne, and Ar (Asplund et al. 2000; Asplund 2000; Allende Prieto et al. 2001, 2002; Asplund et al. 2004 ). These abundance determinations have typical quoted uncertainties of order $0.05 \operatorname{dex}(12 \%)$.

However, as noted earlier the recent lower abundances of these volatile heavy elements, when incorporated into solar models, lead to serious discrepancies with helioseismology. And, to make the situation even more puzzling, the solar models computed with the previously standard heavy element abundances (Grevesse \& Sauval 1998) yield results in good agreement with helioseismology.

Estimating the uncertainty in an abundance determination is even more difficult than arriving at a best-estimate abundance. The important uncertainties are most often systematic and range all the way from line blending in the observed spectra to mathematical and physical approximations made in modeling the solar atmosphere (see Lodders 2003 and Asplund et al. 2005).

We have calculated the neutrino flux dependences on the individual abundances of $\mathrm{C}, \mathrm{N}$, $\mathrm{O}, \mathrm{Ne}, \mathrm{Mg}$, Si, S, Ar and Fe. These are the elements that contribute most to the uncertainty in the calculated fluxes.

With the exception of argon, these are the most abundant metals in the Sun. The elements not included are at least a factor seven less abundant and their uncertainties are small (comparable to the most precise determination of the listed elements). Ar is a special case; its abundance is comparable to that of other elements not listed (for example $\mathrm{Na}, \mathrm{Al}$, $\mathrm{Ca}$ and $\mathrm{Ni}$ ) but the uncertainty in its abundance is much larger (more than four times) and gives a non-negligible contribution to the neutrino fluxes uncertainties despite its low solar abundance.

The neutrino fluxes depend on the individual metal abundances mainly through their effect in the radiative opacities, for which metals are major contributors in the solar interior. In this regard, it is worth mentioning that for each solar model we have produced the set of OPAL radiative opacities (using the tool available at the OPAL Web site ${ }^{1}$ ) corresponding to

\footnotetext{
${ }^{1}$ http://www-phys.llnl.gov/Research/OPAL/new.html
} 
each of the specific compositions used.

\subsection{Conservative and optimistic abundance uncertainties}

We take as one measure of the fractional uncertainty in the heavy element abundance $\beta_{i}$, the difference between the previous standard abundance minus the recently determined abundance divided by the average of the previous abundance and the recently determined abundance. Thus

$$
\frac{\Delta \beta_{i}}{\beta_{i}}=\frac{\left[2\left(\text { Abundance }_{\text {old }, i}-\text { Abundance }_{\text {new }, i}\right)\right]}{\left.\left[\text { Abundance }_{\text {old }, i}+\text { Abundance }_{\text {new }, i}\right)\right]}(1 \sigma) .
$$

Equation (6) represents our 'conservative' estimate of abundance uncertainties and is intended to help take account of systematic uncertainties in an empirical way. We use for the 'old' abundances the recommended Grevesse \& Sauval (1998) values and for the 'new' abundances the values given by Asplund et al. (2005).

We use in equation (6) meteoritic abundances where available, i.e., for $\mathrm{Mg}, \mathrm{Si}, \mathrm{S}$, and Fe. Over the several decades in which photospheric and meteoritic abundances have been compared, the agreement between the photospheric and meteoritic abundances has steadily improved. However, when there was a conflict in the best-estimates from the two methods, it has often turned out that the meteoritic estimate was more accurate. We use solar abundance determinations for $\mathrm{C}, \mathrm{N}, \mathrm{O}, \mathrm{Ne}$, and Ar. The measurements for $\mathrm{Ne}$ and Ar are particularly problematic and subject to systematic uncertainties (see Asplund et al. 2005).

Table 4 gives, in the second column, our preferred (conservative) estimate for the fractional uncertainties of the most important heavy elements. The uncertainties in this column were computed using equation (6) in comparing the Grevesse \& Sauval (1998) abundances with the Asplund et al. (2005) abundances.

The third column of Table 4 gives more optimistic estimates of the composition uncertainties, namely, the uncertainties published in the recent review by Asplund et al. (2005). We list the published uncertainties without taking into account the conflict that the new abundances cause with helioseismology and without taking account of the previous history of the abundance determinations. We interpret conservatively the recently published uncertainties as $1 \sigma$, although the statistical significance of the Asplund et al. (2005) error estimates is not stated explicitly in their paper. We use the meteoritic abundance uncertainties whenever a meteoritic abundance is available; interpreting the meteoritic uncertainties as $1 \sigma$ is especially conservative (Lodders 2003). 
Table 4: Adopted $1 \sigma$ uncertainties for individual heavy elements. We adopt meteoritic measurements where available, i.e., for $\mathrm{Mg}$, Si, S, and Fe (Lodders 2003). For the volatile elements C, N, O, Ne, and Ar we use solar atmospheric abundances. column (2), under the heading 'Historical,' gives as our preferred (conservative) estimated error the fractional differences between the recent abundance determinations (Asplund et al. 2005) and the previously standard values (Grevesse \& Sauval 1998). Column (3), 'Recent Analyses' (optimistic), lists the uncertainties quoted in the recent paper by Asplun et al. (2005); the uncertainties for meteoritic abundances in the Asplund et al. paper are based upon Lodders (2003).

\begin{tabular}{ccc}
\hline \hline $\begin{array}{c}\text { Heavy } \\
\text { Element }\end{array}$ & $\begin{array}{c}\text { Historical } \\
\text { [Conservative] (\%) }\end{array}$ & $\begin{array}{c}\text { Recent Analyses } \\
\text { [Optimistic] (\%) }\end{array}$ \\
\hline $\mathrm{C}$ & 29.7 & 12.2 \\
$\mathrm{~N}$ & 32.0 & 14.8 \\
$\mathrm{O}$ & 38.7 & 12.2 \\
$\mathrm{Ne}$ & 53.9 & 14.8 \\
$\mathrm{Mg}$ & 11.5 & 7.2 \\
$\mathrm{Si}$ & 11.5 & 4.7 \\
$\mathrm{~S}$ & 9.2 & 9.6 \\
$\mathrm{Ar}$ & 49.6 & 20.2 \\
$\mathrm{Fe}$ & 11.5 & 7.2 \\
\hline
\end{tabular}

Comparing the uncertainties given in the second and third columns of Table 4, we see that the recent abundance determinations for the volatile elements have published error estimates (Asplund et al. 2005) that are typical one-third of the uncertainties computed by the historical comparison expressed by equation (6). The uncertainties estimated by Asplund et al. (2005) for the meteoritic determinations are typically a factor of one-half the uncertainties computed with equation (6).

\section{UNCERTAINTIES IN INDIVIDUAL NEUTRINO FLUXES FROM INDIVIDUAL ELEMENT ABUNDANCES}

We define and present in this section the uncertainties in individual neutrino fluxes that result from the uncertainties in each of the important element abundances. Our results are summarized in Table 5 and Table 6. In the discussion, we point out which elements cause the largest uncertainties in the different calculated neutrino fluxes. 
Table 5: Uncertainties of individual neutrino fluxes from individual heavy element abundances. The entries in the table are the fractional uncertainties, $\Delta \phi_{i, j} / \phi_{i}$, of each of the principal solar neutrino fluxes, due to each of the heavy element abundances, $\beta_{j}$, defined by equation (2). The partial derivatives that were used were computed with the aid of the BP04 solar model with the standard composition from Grevesse \& Sauval (1998) and are listed in Table 1. The adopted uncertainties for each heavy element are given in column (2) of Table 4 under the label 'Historical [Conservative].'

\begin{tabular}{|c|c|c|c|c|c|c|c|c|c|}
\hline Source & $\mathrm{C}$ & $\mathrm{N}$ & $\mathrm{O}$ & $\mathrm{Ne}$ & $\mathrm{Mg}$ & $\mathrm{Si}$ & $\mathrm{S}$ & $\mathrm{Ar}$ & $\mathrm{Fe}$ \\
\hline $\mathrm{pp}$ & $-3.63 \mathrm{E}-3$ & $-8.33 \mathrm{E}-4$ & $-1.96 \mathrm{E}-3$ & $-2.15 \mathrm{E}-3$ & $-5.44 \mathrm{E}-4$ & $-1.20 \mathrm{E}-3$ & $-7.04 \mathrm{E}-4$ & $-8.05 \mathrm{E}-4$ & $-2.50 \mathrm{E}-3$ \\
\hline pep & $-6.48 \mathrm{E}-3$ & $-1.66 \mathrm{E}-3$ & $-3.59 \mathrm{E}-3$ & $-2.15 \mathrm{E}-3$ & $-5.44 \mathrm{E}-4$ & $-1.52 \mathrm{E}-3$ & $-1.50 \mathrm{E}-3$ & $-2.41 \mathrm{E}-3$ & $-7.05 \mathrm{E}-3$ \\
\hline hep & $-3.89 \mathrm{E}-3$ & $-1.11 \mathrm{E}-3$ & $-7.50 \mathrm{E}-3$ & $-7.30 \mathrm{E}-3$ & $-1.96 \mathrm{E}-3$ & $-4.02 \mathrm{E}-3$ & $-2.46 \mathrm{E}-3$ & $-2.82 \mathrm{E}-3$ & $-7.48 \mathrm{E}-3$ \\
\hline${ }^{7} \mathrm{Be}$ & $-5.20 \mathrm{E}-4$ & $5.55 \mathrm{E}-4$ & $1.72 \mathrm{E}-2$ & $2.14 \mathrm{E}-2$ & $5.57 \mathrm{E}-3$ & $1.14 \mathrm{E}-2$ & $6.53 \mathrm{E}-3$ & $7.28 \mathrm{E}-3$ & $2.30 \mathrm{E}-2$ \\
\hline${ }^{8} \mathrm{~B}$ & $7.83 \mathrm{E}-3$ & $3.06 \mathrm{E}-3$ & $4.04 \mathrm{E}-2$ & $4.23 \mathrm{E}-2$ & $1.05 \mathrm{E}-2$ & $2.13 \mathrm{E}-2$ & $1.21 \mathrm{E}-2$ & $1.38 \mathrm{E}-2$ & $5.77 \mathrm{E}-2$ \\
\hline${ }^{13} \mathrm{~N}$ & $2.46 \mathrm{E}-1$ & $5.15 \mathrm{E}-2$ & $2.62 \mathrm{E}-2$ & $2.49 \mathrm{E}-2$ & $6.55 \mathrm{E}-3$ & $1.40 \mathrm{E}-2$ & $8.31 \mathrm{E}-3$ & $9.71 \mathrm{E}-3$ & $3.79 \mathrm{E}-2$ \\
\hline${ }^{15} \mathrm{O}$ & $2.40 \mathrm{E}-1$ & $5.97 \mathrm{E}-2$ & $3.09 \mathrm{E}-2$ & $2.98 \mathrm{E}-2$ & $7.65 \mathrm{E}-3$ & $1.65 \mathrm{E}-2$ & $9.64 \mathrm{E}-3$ & $1.13 \mathrm{E}-2$ & $4.46 \mathrm{E}-2$ \\
\hline${ }^{17} \mathrm{~F}$ & $8.62 \mathrm{E}-3$ & $2.78 \mathrm{E}-3$ & $4.34 \mathrm{E}-1$ & $3.33 \mathrm{E}-2$ & $8.53 \mathrm{E}-3$ & $1.80 \mathrm{E}-2$ & $1.06 \mathrm{E}-2$ & $1.26 \mathrm{E}-02$ & $4.95 \mathrm{E}-2$ \\
\hline
\end{tabular}

The fractional uncertainty in each neutrino flux $\phi_{i}$ due to the uncertainty in each element abundance can be computed to high accuracy (Bahcall \& Ulrich 1988, Bahcall 1989) using equation (1)-equation (3). Combining these equations, we can write

$$
\frac{\Delta \phi_{i, j}}{\phi_{i}}=\left[1+\frac{\Delta \beta_{j}}{\beta_{j}}\right]^{\alpha_{i j}}-1 .
$$

The adopted fractional uncertainties $\Delta \beta_{j} / \beta_{j}$ are given in Table 4 and the partial derivatives $\alpha_{i j}$ are given in Table 1 and Table 2 .

Table 5 presents our best estimates of the uncertainties in each solar neutrino flux due to the uncertainty in each element abundance. The results given in Table 5 were evaluated using the partial derivatives computed for the BP04 solar model and given in Table 1; the conservative composition uncertainties are given in the second column of Table 4.

The abundance of iron contributes the largest uncertainty from element abundances for both the ${ }^{7} \mathrm{Be}$ and the ${ }^{8} \mathrm{~B}$ solar neutrino fluxes. Neon is a close second, with oxygen making the third largest contribution.

The iron abundance is relatively well determined (see Table 4) from both photospheric and meteoritic measurements (see Asplund et al. 2005 and Lodders 2003). Nevertheless, the uncertainty from iron is large because of the relatively large partial derivatives of the fluxes with respect to the iron over hydrogen abundance ratio (see Table 1). The oxygen abundance 
Table 6: Optimistic uncertainties of individual neutrino fluxes from individual heavy element abundances, $\phi_{i, j} / \phi_{i}$. The present table is similar to Table 5. However, for the present table we used the more optimistic uncertainties estimated by Asplund et al. (2005); these optimistic uncertainties are listed in column (3) of Table 4 under the label 'Recent Analyses [Optimistic].' Also, we have used here the partial derivatives computed for the solar model BP04+.

\begin{tabular}{|c|c|c|c|c|c|c|c|c|c|}
\hline Source & $\mathrm{C}$ & $\mathrm{N}$ & $\mathrm{O}$ & $\mathrm{Ne}$ & $\mathrm{Mg}$ & $\mathrm{Si}$ & $\mathrm{S}$ & $\mathrm{Ar}$ & $\mathrm{Fe}$ \\
\hline $\mathrm{pp}$ & $-1.15 \mathrm{E}-3$ & $-4.14 \mathrm{E}-4$ & $-5.75 \mathrm{E}-4$ & $-4.14 \mathrm{E}-4$ & $-3.48 \mathrm{E}-4$ & $-4.59 \mathrm{E}-4$ & $-6.41 \mathrm{E}-4$ & $-1.84 \mathrm{E}-4$ & $-1.53 \mathrm{E}-3$ \\
\hline pep & $-2.07 \mathrm{E}-3$ & $-5.52 \mathrm{E}-4$ & $-9.20 \mathrm{E}-4$ & $-2.76 \mathrm{E}-4$ & $-2.09 \mathrm{E}-4$ & $-6.89 \mathrm{E}-4$ & $-1.37 \mathrm{E}-3$ & $-5.52 \mathrm{E}-4$ & $-4.30 \mathrm{E}-3$ \\
\hline hep & $-1.38 \mathrm{E}-3$ & $-4.14 \mathrm{E}-4$ & $-2.07 \mathrm{E}-3$ & $-1.52 \mathrm{E}-3$ & $-1.32 \mathrm{E}-3$ & $-1.79 \mathrm{E}-3$ & $-2.65 \mathrm{E}-3$ & $-9.20 \mathrm{E}-4$ & $-4.99 \mathrm{E}-3$ \\
\hline${ }^{7} \mathrm{Be}$ & $5.76 \mathrm{E}-4$ & $2.76 \mathrm{E}-4$ & $5.31 \mathrm{E}-3$ & $4.57 \mathrm{E}-3$ & $3.97 \mathrm{E}-3$ & $5.30 \mathrm{E}-3$ & $7.36 \mathrm{E}-3$ & $2.21 \mathrm{E}-3$ & $1.61 \mathrm{E}-2$ \\
\hline${ }^{8} \mathrm{~B}$ & $4.04 \mathrm{E}-3$ & $1.24 \mathrm{E}-3$ & $1.15 \mathrm{E}-2$ & $8.87 \mathrm{E}-3$ & $7.47 \mathrm{E}-3$ & $9.78 \mathrm{E}-3$ & $1.38 \mathrm{E}-2$ & $4.24 \mathrm{E}-3$ & $3.92 \mathrm{E}-2$ \\
\hline${ }^{13} \mathrm{~N}$ & $1.02 \mathrm{E}-1$ & $2.52 \mathrm{E}-2$ & $6.35 \mathrm{E}-3$ & $4.98 \mathrm{E}-3$ & $4.53 \mathrm{E}-3$ & $6.40 \mathrm{E}-3$ & $9.39 \mathrm{E}-3$ & $2.76 \mathrm{E}-3$ & $2.50 \mathrm{E}-2$ \\
\hline${ }^{15} \mathrm{O}$ & $9.95 \mathrm{E}-2$ & $2.96 \mathrm{E}-2$ & $7.86 \mathrm{E}-3$ & $5.95 \mathrm{E}-3$ & $5.37 \mathrm{E}-3$ & $7.51 \mathrm{E}-3$ & $1.10 \mathrm{E}-2$ & $3.32 \mathrm{E}-3$ & $2.98 \mathrm{E}-2$ \\
\hline${ }^{17} \mathrm{~F}$ & $4.04 \mathrm{E}-3$ & $1.10 \mathrm{E}-3$ & $1.32 \mathrm{E}-1$ & $6.65 \mathrm{E}-3$ & $6.00 \mathrm{E}-3$ & $8.30 \mathrm{E}-3$ & $1.20 \mathrm{E}-2$ & $3.69 \mathrm{E}-3$ & $3.31 \mathrm{E}-2$ \\
\hline
\end{tabular}

has recently been the subject of very comprehensive studies using the solar atmospheric spectrum. However, it is notoriously difficult to measure the abundance of the noble gases neon and argon, since they are absent in the Fraunhofer absorption line spectrum of the solar photosphere and are mostly lost from meteorites. Measurements of the neon and argon abundance must be made indirectly using coronal data, the solar wind, and energetic solar particles. Since we have limited knowledge of the details of the physics within the regions where neon lines are formed, these indirect measurements are subject to unknown systematic errors.

The abundance of carbon contributes the largest composition uncertainty for the p$\mathrm{p},{ }^{13} \mathrm{~N}$, and ${ }^{15} \mathrm{O}$ solar neutrino fluxes. In fact, the uncertainty in the carbon abundance dominates the composition uncertainty for the ${ }^{13} \mathrm{~N}$ and ${ }^{15} \mathrm{O}$ neutrino fluxes.

Table 6 presents more optimistic estimates for the uncertainties in the individual neutrino fluxes from individual heavy elements. These smaller uncertainties are calculated using the abundance uncertainties given by Asplund et al. (2005) (column [3] of Table 4) and the partial derivatives computed using the BP04+ solar model (Table 2).

The largest composition uncertainty in the calculation of the ${ }^{7} \mathrm{Be}$ and ${ }^{8} \mathrm{~B}$ neutrino fluxes is again the iron abundance given the assumptions used in calculating Table 6 , just as it was for the more conservative uncertainty estimates used in constructing Table 5. However, for the assumptions used in calculating Table 6, sulphur contributes the second largest 
uncertainty and oxygen, neon, and silicon all contribute significantly.

For the p-p neutrino flux, the largest uncertainty due to composition is from the iron abundance (for the conditions of Table 6). But, for the ${ }^{13} \mathrm{~N}$ and ${ }^{15} \mathrm{O}$ neutrino fluxes, the carbon abundance dominates the uncertainty due to composition.

Why do we use for Table 6 the partial derivatives computed with the BP04+ model? Since we use in Table 6 the uncertainties determined by recent measurements, it is more appropriate to use the partial derivatives that are obtained with a model, BP04+, that adopts the recent abundance determinations. The precise composition used in constructing BP04+ is given in Table 1 of Bahcall et al. (2004b). There are slight differences between the composition used in constructing $\mathrm{BP} 04+$ and the very latest estimated abundances given by Asplund et al. (2005). To make sure that these small differences were unimportant, we evolved a solar model that was identical to BP04 and BP04+ except that the new model, BP04AGS, uses the Asplund et al. (2005) abundances. The average difference between the total neutrino flux uncertainties due to composition uncertainties that was obtained using models BP04+ and BP04AGS, was $0.09 \%$ and, in all cases, less than $0.2 \%$.

\section{NEUTRINO FLUX AND EXPERIMENTAL RATE UNCERTAINTIES FROM ALL ABUNDANCE UNCERTAINTIES}

We begin this section by describing in $\S 5.1$ how we combine all of the abundance uncertainties to compute the total uncertainty in the calculated neutrino fluxes that arise from all composition determinations, assuming that the abundance determinations of different elements are statistically independent. We discuss in $\S 5.2$ the special case of the neon and argon abundances, which are determined relative to a reference element that can be measured in the solar photosphere. We discuss in $\S 5.3$ the correlated uncertainty in the meteoritic abundances that results from adjusting the meteoritic abundance scale to agree with the solar atmospheric abundance scale. We compare in $\S 5.4$ the conservative uncertainty estimates (our preferred estimates) with the more optimistic uncertainty estimates that are based upon the published error estimates of abundance uncertainties by Asplund et al. (2005). In $\S 5.5$, we compare the uncertainties that have been traditionally estimated using the total $Z / X$ with the uncertainties that are estimated using individual abundance uncertainties.

Table 7 summarizes the principal results of this section.

The software used to combine the abundance uncertainties is available at

http://www.sns.ias.edu/ jnb under the menu items Solar Neutrinos/software and data. The 
code, exportrates.f, provides options for calculating the uncertainties using individual abundance uncertainties and also using the uncertainty in the total $Z / X$.

\subsection{Computation of flux uncertainties from all abundance uncertainties}

The uncertainty due to abundance determinations for a given calculated neutrino flux can be obtained by combining the effects of all the element uncertainties on the flux of interest. If all of the abundance determinations are independent of each other, the uncertainties can be combined quadratically. However, if there are correlations in the uncertainties in the abundance determinations, these must be taken into account. Usually, observers do not specify the correlations among the quoted uncertainties. However, as we discuss in $\S 5.2$, there is a strong correlation between the uncertainties in the neon and argon abundances and in the oxygen abundance and we take this into account.

The general formula for the uncertainty in the neutrino flux $\phi_{i}$ can be written

$$
\frac{\Delta \phi_{i}}{\phi_{i}}=\sqrt{\sum_{j}\left(\frac{\Delta \phi_{i, j}}{\phi_{i}}\right)^{2}+\sum_{k \neq l}\left(\frac{\Delta \phi_{i, k}}{\phi_{i}}\right)\left(\frac{\Delta \phi_{i, l}}{\phi_{i}}\right) \rho(k, l)},
$$

where the indices $j, k$, and $l$ denote different elements and $\rho(k, l)$ is the correlation coefficient between the abundance uncertainties of the $k$ and $l$ elements. If the uncertainties of two elements, $k$ and $l$, are uncorrelated then $\rho(k, l)=0.0$. If the uncertainties are fully correlated, $\rho(k, l)=1.0$.

For radiochemical experiments, chlorine, gallium, and lithium experiments, the procedure for calculating the uncertainty, $\Delta R$, in predicted event rates, $R$, is somewhat more complicated. The measured rates for radiochemical experiments are sensitive to contributions from different neutrino branches, with each neutrino flux contributing an amount $\phi_{i} \sigma_{i}$ (where $\sigma_{i}$ is the neutrino absorption cross section). The uncertainty from a given element abundance, $\beta_{j}$, affects in a coherent way to all of the partial contributions $\phi_{i} \sigma_{i}$. The contribution to the uncertainty in the rate from a fixed composition $j, \Delta r(j)$, is

$$
\Delta r(j)=\sum_{i} \phi(i) \sigma(i)\left(\frac{\Delta \phi_{i, j}}{\phi_{i}}\right) .
$$

To calculate the uncertainty for a radiochemical rate with only incoherent contributions, we first sum over all partial rate contributions $i$ for a fixed composition uncertainty $j$, before quadratically combining the uncertainties from each different composition $j$. 
If the uncertainties from some elements are correlated, then we must include the effects of the correlations as an additional term. Including the possibility of correlations, the general expression for the uncertainty in a radiochemical experiment is

$$
\Delta \text { Rate }=\sqrt{\sum_{j}(\Delta r(j))^{2}+\sum_{k \neq l} \Delta r(k) \Delta r(l) \rho(k, l)} .
$$

\subsection{Correlation of neon, argon, and oxygen abundance uncertainties}

The noble gases neon and argon do not appear in the solar photospheric spectrum and are largely lost by meteorites. Therefore, the abundances of neon and argon abundances must be determined in environments that are less well understood than the photospheric spectrum, in particular, in the coronal spectrum, in the solar wind, in solar energetic particles, and by gamma ray spectroscopy. The measurements of the neon and argon abundances must be made with respect to some reference element that does appear in the solar photosphere. The reference element of choice is usually oxygen or magnesium. There are, of course, potentially very large systematic uncertainties in these indirect determinations of the neon and argon abundances.

Asplund et al. (2005) give neon and argon abundances that are determined relative to the oxygen abundance. Thus the uncertainties in the neon and argon abundances are correlated with the uncertainty in the oxygen abundance. From the uncertainties given in Table 1 of Asplund et al. (2005), we infer that Asplund et al. believe that the uncertainty in the neon abundance is dominated by the uncertainty in the oxygen abundance and that the uncertainty in the argon abundance is due to comparable contributions from the measurement of the oxygen abundance and from the $\mathrm{Ar} / \mathrm{O}$ ratio.

We have made calculations based upon two extreme assumptions. First, we assume that all the abundance determinations, including those of oxygen, neon, and argon, are independent. Second, we assume that the uncertainties in the oxygen, neon, and argon abundances are completely correlated. Given this second assumption, we set $\rho(k, l)=1.0$ in equation (8) and equation (10) when both $k$ and $l$ represent either $\mathrm{O}, \mathrm{Ne}$, or Ar (otherwise, we take $\rho(k, l)=0.0)$.

Fortunately, the differences in the flux uncertainties are relatively small when the uncertainties are calculated in these two extreme ways. The average fractional difference in a neutrino flux uncertainty due to assuming complete correlation (of the $\mathrm{O}$, Ne, and Ar uncertainties) or no correlation varies between $6 \%$ and $17 \%$ of the composition uncertainty itself, depending upon which partial derivatives (BP04 or BP04+) are used and depending upon 
whether we adopt conservative or optimistic uncertainties for the element abundances. Since the composition uncertainty is only one of a number of different sources of flux uncertainty (see discussion in $\S 6$ ), a $17 \%$ uncertainty in the composition uncertainty is acceptable. We certainly do not believe the composition uncertainties discussed in $\S 3$ and Table 4 are reliable to $17 \%$ of the quoted uncertainty. After all, the observers do not as a rule specify the confidence level which their uncertainties represent (see, however, the Lodders 2003 analysis of meteoritic abundances).

In compiling Table 7, we again made a conservative assumption, namely, that the oxygen, neon, and argon uncertainties are completely correlated. Since all three elements have, for a given neutrino flux, the same sign for their partial derivatives (see Table 1 and Table 2), this procedure results in a larger uncertainty than if we had combined incoherently the oxygen, neon, and argon uncertainties. The assumption we make here slightly overestimates the total composition uncertainty assuming the correctness of all the other numbers that go into the analysis.

In future compilations of solar abundances and their uncertainties, it will be very useful if the compilers specify the correlation between the difference abundance uncertainties.

\subsection{Correlations of meteoritic abundances via a scale factor}

Traditionally, the solar abundances of the elements that can be measured in the solar photosphere or elsewhere in the solar atmosphere are determined relative to the abundance of hydrogen. However, hydrogen is lost from meteorites. Therefore, the abundances that are measured in meteorites are determined relative to some other element, which is usually taken as Si. The two scales, the atmospheric and the meteoritic, are adjusted to give a consistent set of values by sliding one of the scales up or down with respect to the other scale. For specificity, we can think of this procedure as adjusting the meteoritic scale with respect to the atmospheric scale.

The uniform adjustment of the meteoritic scale implies that there is a correlation of all of the meteoritic abundances among themselves. The amount of this correlation can be estimated by calculating how much we have to change the meteoritic scale with respect to the atmospheric scale in order to significantly affect the goodness of the agreement between the two scales, which is generally excellent (see Grevesse \& Sauval 1998, Lodders 2003, Asplund et al. 2005). We therefore need to evaluate

$$
\sigma_{\text {meteorite, atmosphere }}=\sqrt{\frac{1}{N} \sum_{i}\left(\beta_{\text {meteorite }, i}-\beta_{\text {atmosphere }, i}\right)^{2}}
$$


where $\beta_{\text {meteorite }, i}$ and $\beta_{\text {atmosphere }, i}$ are, respectively, the meteoritic and atmospheric abundances of the element $i$.

We are interested in the accuracy with which the meteoritic and atmospheric scales can be brought into agreement for the abundant heavy elements, since it is only the abundant heavy elements that affect the neutrino fluxes significantly through their contributions to the radiative opacity. Moreover, it is plausible that the relative abundances are more robust for elements that are more abundant.

We have evaluated the correlation that is expressed in equation (11) for a number of different cases. We find that the correlation is small and the general size of the correlation is robust. For example, we have computed the correlation $\sigma_{\text {meteorite, atmosphere for the seven }}$ elements with logarithmic abundances relative to hydrogen that are greater than 6.0 on the usual scale in which the hydrogen abundance is set equal to 12.0 . For the Lodders (2003) abundances, the unweighted average is $\sigma_{\text {meteorite, atmosphere }}=0.7 \%$ and the weighted average (quadratically combined atmospheric and meteoritic errors) is $\sigma_{\text {meteorite, atmosphere }}=0.9 \%$. These results are essentially unchanged if we throw out, e.g., the Ni abundance, which is the least well determined of the sample we are considering. Similar results are obtained for the Asplund et al. (2005) abundances. We find an unweighted average of $\sigma_{\text {meteorite, atmosphere }}=$ $1.8 \%$ and a weighted average of $2.1 \%$.

The correlated contribution of the uncertainty due to the relative adjustment of the meteoritic and atmospheric scales depends quadratically upon $\sigma_{\text {meteorite, atmosphere. It follows }}$ from the definition of the correlation coefficient $\rho(k, l)$ between the uncertainties of the meteoritic abundances of two elements $k \neq l$ that

$$
\rho(k, l)=\frac{\sigma_{k, l}}{\sigma_{k} \sigma_{l}}
$$

where $\sigma_{k, l}$ is the covariance between the uncertainties for the two elements and $\sigma_{k}$ and $\sigma_{l}$ are the uncertainties of each element abundance. The individual uncertainties, $\sigma_{k}$ and $\sigma_{l}$, include the quadratically-combined meteoritic measurement uncertainty plus the covariance from the scale adjustment.

We do not have a good way of calculating the covariance, but we hope that the covariance will be evaluated in the future by the authors of papers presenting critical summaries of solar element abundances. We can obtain a reasonable upper limit to the covariance by assuming

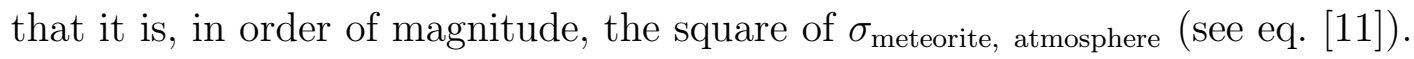

The precise values of $\rho(k, l)$ depend upon whose compilation of solar abundances one uses and upon how one defines the sample of abundant heavy elements within the published list of abundances. However, in order of magnitude, $\sigma_{\text {atmospheric, }} \sim \sigma_{\text {meteoritic }} \sim 0.1$ and the characteristic value of $\sigma_{k, l} \sim 0.01$. Hence, $\rho(k, l) \sim 0.01$. 
Since $\rho$ is small, we can neglect, without making a significant numerical error, the effects of the relative adjustment of the meteoritic and atmospheric abundance scales in equation (8) and equation (10). This approximation is satisfactory even though there are seven times as many off-diagonal terms as diagonal terms, .

In the future, when there is general agreement on the correct solar abundances and their uncertainties, the effect of the relative adjustment of the meteoritic and atmospheric scales can be taken into account using equations (8), (10), and (11).

\subsection{Comparison of conservative and optimistic uncertainty estimates}

Table 7 presents our estimates for the total uncertainty in each calculated neutrino flux and in each radiochemical rate due to all composition sources. We present in the table the results from a conservative estimate (column [2]), an optimistic estimate (column [3]), and the traditional method that uses the total $Z / X$. The entries in the table were computed using equation (8) and equation (10), except that we have combined coherently the uncertainties in the oxygen, neon, and argon abundances as described in $\S 5.2$.

The second column of Table 7 presents our best estimate for the total uncertainty from all composition uncertainties for each neutrino flux and for the rate of each radiochemical experiment. The partial derivatives used in these calculations were taken from Table 1 (solar model BP04) and, for the entries enclosed in parentheses, Table 2 (solar model BP04+). We also used for the second column of Table 7 the conservative individual abundance uncertainties listed in the second column of Table 4.

We present the much more optimistic estimates of the total composition uncertainties in the third column of Table 7. The entries in the third column were calculated using the abundance uncertainties (Asplund et al. 2005) that are listed in the third column of Table 4. We also used the BP04+ partial derivatives from Table 2.

The conservative composition uncertainties listed in the second column of Table 7 are typically a factor of two or more larger than the more optimistic composition uncertainties listed in the third column of the table.

\subsection{Uncertainties calculated using total $Z / X$}

The last (fourth) column of Table 7 lists the composition uncertainties that are computed by using the traditional method of lumping together all heavy elements, i.e., using 
Table 7: Neutrino Flux and Rate Uncertainties from All Solar Composition Uncertainties. The abundance uncertainties used in calculating the entries in the second column of the present table were obtained using the historical (conservative) composition uncertainties that are listed in the second column of Table 4; the uncertainties from recent analyses (our 'optimistic' uncertainties) are given in the third column of Table 4 and were used to obtain the entries in the third column of the present table. The uncertainties without parentheses that are listed in columns (2) and (3) of the table were calculated using partial derivatives from Table 1 that were obtained with solar models that had the BP04, i.e., the 1998 Grevesse \& Sauval solar composition of heavy elements. The uncertainties in parentheses that are listed in columns (2) and (3) were calculated with partial derivatives from Table 2 that were obtained with solar models that had the BP04+ recently-determined solar composition (see Table 1 of Bahcall, Serenelli, \& Pinsonneault 2004). The uncertainties in column (4) were calculated assuming that (see Bahcall \& Pinsonneault 2004) the total spread in all modern measurements of the heavy element abundance by mass divided by the hydrogen abundance by mass, $Z / X$, is equal to the $3 \sigma$ uncertainty in $Z / X$, i.e., $\Delta(Z / X) /(Z / X)=0.15(1 \sigma)$. For column (4), the values without parentheses were calculated by Bahcall \& Pinsonneault (2004) with the Bahcall \& Ulrich (1988) partial derivatives and the values with parentheses were calculated with the BP04 partial derivatives (see Table 3).

\begin{tabular}{lccc}
\hline \hline $\begin{array}{l}\text { Neutrino } \\
\text { Flux }\end{array}$ & $\begin{array}{c}\text { Historical } \\
\text { (Conservative) }\end{array}$ & $\begin{array}{c}\text { Recent Analyses } \\
\text { (Optimistic) }(\%)\end{array}$ & $\begin{array}{c}Z / X: \\
\text { Historical }(\%)\end{array}$ \\
\hline $\mathrm{pp}$ & $0.7(0.5)$ & $0.3(0.2)$ & $1.0(1.0)$ \\
pep & $1.3(1.0)$ & $0.6(0.5)$ & $2.0(2.1)$ \\
hep & $2.0(1.6)$ & $0.9(0.8)$ & $2.6(2.9)$ \\
${ }^{7} \mathrm{Be}$ & $5.3(4.6)$ & $2.4(2.2)$ & $8.0(8.6)$ \\
${ }^{8} \mathrm{~B}$ & $11.6(9.9)$ & $5.3(5.0)$ & $20.0(22.0)$ \\
${ }^{13} \mathrm{~N}$ & $26.2(25.8)$ & $11.1(11.0)$ & $33.2(34.1)$ \\
${ }^{15} \mathrm{O}$ & $26.2(25.7)$ & $11.2(11.0)$ & $37.5(38.2)$ \\
${ }^{17} \mathrm{~F}$ & $48.3(45.4)$ & $15.6(14.7)$ & $39.1(41.3)$ \\
\hline $\mathrm{Experiment}$ & $\mathrm{SNU}$ & $\mathrm{SNU}$ & $\mathrm{SNU}$ \\
\hline${ }^{37} \mathrm{Cl}$ & $0.9(0.8)$ & $0.4(0.4)$ & $1.6(1.7)$ \\
${ }^{71} \mathrm{Ga}$ & $4.4(4.0)$ & $1.9(1.9)$ & $8.2(8.7)$ \\
${ }^{7} \mathrm{Li}$ & $5.7(5.3)$ & $2.5(2.5)$ & $10.6(11.2)$ \\
\hline
\end{tabular}

the partial derivatives $\partial \ln \phi_{i} / \partial \ln (Z / X)$ (see eq. [5]). In this calculation, we assumed that $\Delta(Z / X) /(Z / X)=0.15(1 \sigma)$, based upon the historical time dependence of the published values of $Z / X$ (Bahcall \& Pinsonneault 2004).

The traditional total $Z / X$, or historical, method (last column of Table 7 ) yields (except for the $\mathrm{C}, \mathrm{N}, \mathrm{O}$ neutrino fluxes) composition uncertainties that vary from about $50 \%$ larger 
to a factor of two larger than the conservative uncertainties (column (2) of Table 7) estimated by our preferred method that is based upon individual abundances (second column of Table 7 ). The two most important special cases are the ${ }^{7} \mathrm{Be}$ and the ${ }^{8} \mathrm{~B}$ neutrino fluxes for which the traditional $Z / X$ method gave uncertainties of $8.0 \%$ and $20.0 \%$, respectively. Our best estimates (conservative) using errors on individual abundance determination are $5.3 \%$ and $11.6 \%$ for these same two neutrino fluxes. For the $\mathrm{CNO}$ neutrino fluxes, ${ }^{13} \mathrm{~N},{ }^{15} \mathrm{O}$, and ${ }^{17} \mathrm{~F}$, the estimated total uncertainties are comparable for both ways of computing the total uncertainties (individual abundances and a single $Z / X$ ).

The total composition uncertainties for the ${ }^{37} \mathrm{Cl},{ }^{71} \mathrm{Ga}$, and ${ }^{7} \mathrm{Li}$ radiochemical solar neutrino experiments are reduced by about a factor of two by taking account of the sensitivities to individual compositions.

\section{UNCERTAINTIES FROM ALL KNOWN SOURCES}

We present in $\S 6.1$ our estimates for the total uncertainties in the solar neutrino fluxes calculated with standard solar models. We present uncertainties obtained with our preferred conservative approach, with a more optimistic approach, and with the traditional $Z / X$ approach. We compare and discuss in $\S 6.2$ the total uncertainties obtained by different methods.

\subsection{Total uncertainties in neutrino fluxes from all sources}

The uncertainties in neutrino fluxes and rates due to all the known sources of uncertainties, including nuclear reaction rates, radiative opacity, element diffusion, the solar luminosity as well as element abundances, can be computed using equation (8) and equation (10). Each separate source of uncertainty is represented by an index $j$ in these equations. We adopt the uncertainties for all of the sources except element abundances as presented in the recent discussion by Bahcall \& Pinsonneault (2004).

The uncertainties due to radiative opacity and element diffusion deserve special attention, since they could in principle be affected by the recent abundance determiantions. The uncertainty due to radiative opacity has been calculated by comparing the neutrino fluxes computed with solar models that used the older Los Alamos and the the much-improved and (then) new Livermore opacities, the first and third rows of Table 7 of Bahcall and Pinsonneault (1992). The fractional differences in the calculated neutrino fluxes were taken to be $3 \sigma$. We expect that the estimated uncertainties due to radiative opacities will be re- 
Table 8: Neutrino Flux Uncertainties from all known sources. The uncertainties listed in the table include all known sources of uncertainties (see Bahcall \& Pinsonneault 2004). The labels of the columns have the same meaning as in Table 7. Columns (2)-(4) differ only in the way the uncertainties due to the solar heavy element abundances were calculated. However, each entry in the present table includes the quadratically combined total uncertainties from all sources rather than just the uncertainties from the composition as given in Table 7.

\begin{tabular}{lccc}
\hline \hline $\begin{array}{l}\text { Neutrino } \\
\text { Flux }\end{array}$ & $\begin{array}{c}\text { Historical } \\
\text { (conservative) }(\%)\end{array}$ & $\begin{array}{c}\text { Recent Analyses } \\
\text { (optimistic) }(\%)\end{array}$ & $\begin{array}{c}Z / X: \\
\text { Historical }(\%)\end{array}$ \\
\hline pp & $1.0(0.9)$ & $0.8(0.8)$ & $1.2(1.3)$ \\
pep & $1.7(1.5)$ & $1.3(1.2)$ & $2.3(2.3)$ \\
hep & $15.5(15.5)$ & $15.4(15.4)$ & $15.6(15.6)$ \\
${ }^{7} \mathrm{Be}$ & $10.5(10.1)$ & $9.3(9.3)$ & $12.1(12.5)$ \\
${ }^{8} \mathrm{~B}$ & $16.3(15.1)$ & $12.6(12.5)$ & $23.0(24.8)$ \\
${ }^{13} \mathrm{~N}$ & ${ }_{-28.1}^{+31.2}\left({ }_{-27.8}^{+30.9}\right)$ & ${ }_{-15.1}\left({ }_{-15.1}^{+20.2}\right)$ & ${ }_{-34.8}^{+37.3}\left(_{-35.6}^{+38.1}\right)$ \\
${ }^{15} \mathrm{O}$ & ${ }_{-28.8}^{+33.2}\left({ }_{-28.4}^{+32.8}\right)$ & $\left.{ }_{-16.4}^{+23.3}{ }_{-16.4}^{+23.2}\right)$ & ${ }_{-39.4}^{+42.7}\left({ }_{-40.1}^{+43.3}\right)$ \\
${ }^{17} \mathrm{~F}$ & $52.2(49.5)$ & $25.1(24.5)$ & $43.8(45.7)$ \\
\hline Experiment & $\mathrm{SNU}$ & $\mathrm{SNU}$ & $\mathrm{SNU}$ \\
\hline${ }^{37} \mathrm{Cl}$ & $1.3(1.2)$ & $1.0(1.0)$ & $1.8(1.9)$ \\
${ }^{71} \mathrm{Ga}$ & ${ }_{-9.5}^{+9.5}\left({ }_{-9.3}^{+9.4}\right)$ & ${ }_{-8.6}^{+8.7}\left({ }_{-8.6}^{+8.7}\right)$ & ${ }_{-11.7}^{+11.8}\left({ }_{-12.1}^{+12.2}\right)$ \\
${ }^{7} \mathrm{Li}$ & ${ }_{-7.2}^{+7.6}\left({ }_{-7.0}^{+7.3}\right)$ & ${ }_{-5.1}^{+5.6}\left({ }_{-5.1}^{+5.6}\right)$ & ${ }_{-11.5}^{+11.7}\left({ }_{-12.1}^{+12.3}\right)$ \\
\hline
\end{tabular}

duced somewhat when a similar comparison is made between the Livermore opacities and the recently published OPAL opacities (Badnell et al. 2004, Seaton \& Badnell et al. 2004).

The uncertainties in the neutrino fluxes due to element diffusion are calculated in three steps. First, a solar model was constructed with full element diffusion and then a similar model was constructed with no diffusion (see Model 9 and Model 10 Table III of Bahcall \& Pinsonneault 1995). Next, the fractional differences in the neutrino fluxes between the two models are formed. These differences represent the extreme changes between no diffusion and our best estimate for diffusion. Finally, the fractional differences are multiplied by $15 \%$, which is the $3 \sigma$ error estimate on the calculation of the diffusion rate made in the original 
paper of Thoul, Bahcall, and Loeb $(1994)^{2}$

Table 8 presents the total uncertainties from all sources. The structure of Table 8 is similar to Table 7. For both tables, the column labeled 'Historical' was calculated by interpreting as $1 \sigma$ uncertainties the difference between the best-estimates for element abundances given by Grevesse \& Sauval (1998) and by Asplund et al. (2005). For the third column, labeled 'Recent Analyses,' we adopted the abundance uncertainties given in the recent review by Asplund et al. (2005). The last column, labeled ' $Z / X$ : Historical' assumes that $\Delta(Z / X) /(Z / X)=0.15(1 \sigma)$. The entries in columns (2) and (3) without parentheses (in parentheses) were calculated using logarithmic partial derivatives with respect to the BP04 (BP04+) solar models. The entries without parentheses in the last column, ' $Z / X$ : Historical,' were calculated by Bahcall \& Pinsonneault (2004) using the logarithmic partial derivatives of Bahcall \& Ulrich (1988); the entries in parentheses in column (4) were calculated with partial derivatives obtained using the BP04 solar model.

\subsection{Comparison of total uncertainties obtained with different assumptions}

Our preferred, most conservative estimates are given in column (2) of Table 8 and should be compared with the more optimistic estimates of uncertainties given in column (3). The total uncertainty in the ${ }^{8} \mathrm{~B}$ neutrino flux is most affected by the difference between the two methods of calculating the composition uncertainties. The conservative approach yields a $16.3 \%$ uncertainty for the calculated ${ }^{8} \mathrm{~B}$ solar neutrino flux, while the optimistic approach yields a $12.5 \%$ uncertainty, which is $30 \%$ smaller.

The traditional (total $Z / X)$ method of estimating the uncertainty from the solar composition yields a much larger value for the total uncertainty, 23.0\% (24.8\% with BP04 partial derivatives used for both cases). The $Z / X$ method therefore overestimates the total uncertainty from all sources by $41 \%$ (51\% using BP04 partial derivatives for both cases) and by a factor of two relative to the optimistic individual composition uncertainties.

The ${ }^{8} \mathrm{~B}$ neutrino flux is extremely rare but also extremely important. The SNO (Ahmed et al. 2004) and Super-Kamiokande (Fukuda et al. 2001) solar neutrino experiments measure

\footnotetext{
${ }^{2}$ We calculated solar models with the Asplund et al. (2005) composition but with a diffusion coefficient changed by $\pm 15 \%$ from the best-estimate of Thoul et al. (1994). The many-sigma disagreements with the helioseismology persist. There are moderate improvements in the agreement with some of the quantities but moderate increases in the disagreements with other quantities. We conclude that adjustments in the diffusion coefficient can not resolve the discrepancy between solar model predictions and helioseismological measurements if the Asplund et al. (2005) abundances are adopted.
} 
only ${ }^{8} \mathrm{~B}$ neutrinos and the chlorine radiochemical experiment (Cleveland et al. 1998) is primarily sensitive to ${ }^{8} \mathrm{~B}$ neutrinos. The current accuracy of the experimental measurement of the ${ }^{8} \mathrm{~B}$ solar neutrino flux is about $9 \%$ (Ahmed et al. 2004), which is much less than the theoretical uncertainty. Moreover, the uncertainty in the predicted ${ }^{8} \mathrm{~B}$ neutrino flux is an important parameter in many analyses of neutrino oscillation characteristics that make use of solar neutrino measurements.

For the p-p, pep, hep, and ${ }^{7}$ Be solar neutrino fluxes, the difference in the total neutrino uncertainties between the conservative error estimates (column [2] of Table 4) and the more optimistic error estimates (column [3] of Table 4) are not large enough to affect the interpretation of planned or ongoing solar neutrino experiments. Even the total $Z / X$ method gives estimates for the total flux uncertainties that are similar to the results obtained by conservative or optimistic assumptions about the individual abundance uncertainties.

The most abundant neutrino fluxes from the CNO reactions, the ${ }^{13} \mathrm{~N}$ and ${ }^{15} \mathrm{O}$ neutrino fluxes, are very roughly proportional to the assumed CNO abundances. Since the recently published abundance analyses of the volatile elements (including $\mathrm{C}, \mathrm{N}$, and $\mathrm{O}$ ) are much lower than previously believed, but with a current quoted error that is relatively small, the total uncertainties for the CNO neutrino fluxes are about a factor of two larger when computed using the total $Z / X$ method than the uncertainties that are obtained using the recent analysis of abundance uncertainties by Asplund et al. (2005). Unfortunately, no precision experiments to measure the CNO solar neutrinos are currently planned.

If we had combined the $\mathrm{O}, \mathrm{Ne}$, and Ar abundance uncertainties incoherently rather than coherently as was done in constructing Table 8 (see discussion in $\S 5.2$ ), the total conservative uncertainty in the ${ }^{8} \mathrm{~B}$ neutrino flux would have been $14.4 \%$ instead of our preferred value of 16.3\%. The differences between the coherent and incoherent combination of the $\mathrm{O}, \mathrm{Ne}$, and Ar abundance uncertainties are much less important for all the other solar neutrino fluxes (less than $10 \%$ of the total estimated uncertainty, a change which is beyond the anticipated experimental precision).

\section{SUMMARY AND DISCUSSION}

In this paper, we have computed for the first time the sensitivity of each solar neutrino flux to the abundance of each chemical element on the surface of the Sun. With these computations, we are able to identify which element abundances most strongly affect the solar neutrino fluxes. Moreover, we are able to calculate a better-founded estimate of the total uncertainty in each neutrino flux due to composition uncertainties. In previous treat- 
ments, all the uncertainties in the solar chemical composition have been lumped into a single parameter, the heavy element to hydrogen ratio, $Z / X$.

The results presented here are important for solar neutrino research since the flux that is most affected by our more detailed error treatment, the ${ }^{8} \mathrm{~B}$ solar neutrino flux, is also the flux that is measured directly by the SNO and the Super-Kamiokande solar neutrino experiments.

We summarize in $\S 7.1$ our results for the partial derivatives of each neutrino flux with respect to each element abundance. We also remark in this subsection on the historical robustness of the partial derivatives $\partial \ln \phi / \partial \ln (Z / X)$. We then describe in $\S 7.2$ our conservative and optimistic estimates for uncertainties in the individual element abundances. We summarize our principal results in $\S 7.3$. We first discuss which individual element abundances contribute most to the neutrino flux uncertainties $(\S 7.3 .1)$ and then we describe the results when the uncertainties from all elements are combined ( $\S 7.3 .2$ ). Finally, we summarize the uncertainties from all known sources of error ( $\S 7.3 .4$ ). We present in $\S 7.5$ our final word on the subject of solar abundances and neutrino fluxes.

\subsection{Partial derivatives}

The principal calculational tool in our analysis is the set of partial derivatives, $\alpha_{i j}$, of each solar neutrino flux with respect to each element abundance. These partial derivatives are defined in equation (1) and equation (2) and are presented in Table 1 and Table 2.

Throughout this paper, we present estimates for neutrino flux uncertainties that use the partial derivatives obtained with the aid of two different solar models, BP04 (which incorporates the older Grevesse \& Sauval 1998 solar abundances) and BP04+ (which incorporates recent abundance determinations for the volatile elements, see Table 1 of Bahcall et al. 2004b). The tabulated results in this paper show that the estimated uncertainties in the neutrino fluxes are essentially the same whether the partial derivatives $\alpha_{i j}$ are calculated using the solar model BP04 or the solar model BP04+. In the tables in the main text, we present without parentheses (with parentheses) the results obtained using the BP04 solar model (the BP04+) solar model.

For comparison with earlier results and for testing the robustness of partial derivative calculations over more than two decades, we present in Table 3 published values from 1982, 1988 , and 2004 for the partial derivatives with respect to $Z / X$. The rms fractional change in the partial derivatives $\partial \ln \phi / \partial \ln (Z / X)$ is only $2 \%$ when the partial derivatives of Bahcall \& Ulrich (1988) are compared with those obtained using the recent solar model BP04. Despite 
all the improvements of the solar model since 1988, the partial derivatives are practically unchanged.

Table 7 and Table 8 compare in their last columns the uncertainties computed with the 1988 partial derivatives $\partial \ln \phi / \partial \ln (Z / X)$ and with the 2004 partial derivatives. The total uncertainties due to abundances and the total uncertainties due to all sources are practically the same when computed with the old and new derivatives.

\subsection{Abundance uncertainties: conservative and optimistic}

We estimate conservatively the uncertainty in the element abundances by comparing the previously standard (Grevesse \& Sauval 1998) and the most recent abundance determinations (Asplund et al. 2005). We adopt as $1 \sigma$ the differences between the previous and the recent abundance determinations; this conservative estimate is our preferred assessment of the abundance uncertainties. Asplund et al. (2005) give, based upon recent analyses, abundance uncertainties that are smaller than our conservative estimates. We adopt as our optimistic estimate of the abundance uncertainties the results given by Asplund et al. (2005).

Table 4 summarizes the adopted conservative and optimistic uncertainties for the individual element abundances.

We perform all of our calculations with both the conservative and with the optimistic estimates for the abundance uncertainties and compare the results obtained with both sets of abundance uncertainties.

\subsection{Results}

\subsubsection{Flux uncertainties due to individual element abundances}

We present in Table 5 and Table 6 the uncertainty in each neutrino flux due to uncertainties in the determination of each of the most important chemical elements. The largest uncertainty for both the important ${ }^{7} \mathrm{Be}$ and ${ }^{8} \mathrm{~B}$ neutrino fluxes is due to the iron abundance, which is strongly ionized in the solar interior where the neutrinos are produced. Oxygen, neon, silicon, and sulphur all contribute significantly to the ${ }^{7} \mathrm{Be}$ and ${ }^{8} \mathrm{~B}$ neutrino flux uncertainties.

The neon abundance is particularly problematic since it cannot be measured directly in the solar photosphere and escapes from meteorites. The abundance uncertainties estimated 
for neon may be regarded as best-guesses based upon our limited understanding of the environments in which neon is detectable in the Sun.

For the ${ }^{13} \mathrm{~N}$ and ${ }^{15} \mathrm{O}$ solar neutrino fluxes, the carbon abundance dominates the composition uncertainty. Carbon is also the largest contributor to the calculated composition uncertainty of the basic p-p solar neutrino flux if we adopt the conservative abundance uncertainties. For the more optimistic abundance uncertainties of Asplund et al. (2005), iron contributes somewhat more than carbon to the estimated uncertainty in the p-p neutrino flux.

\subsubsection{Combined flux uncertainties from all elements}

Table 7 gives for each solar neutrino flux the total uncertainty from all the abundance determinations. We have used equation (8) to combine the uncertainties from different chemical elements except for oxygen, neon, and argon. We combine coherently the abundance uncertainties from oxygen, neon, and argon (see discussion in $\S 5.2$ ), since the abundances of neon and argon are measured with respect to oxygen. Our preferred (conservative) estimated uncertainties are given in the second column of Table 7, whereas the more optimistic uncertainties are presented in column (3). The conservative uncertainties are, in all cases, more than a factor of two larger than the optimistic uncertainties.

We present in the fourth column of Table 7 the larger uncertainties estimated using the traditional lumping-together of all abundance uncertainties into a single $\Delta(Z / X) /(Z / X)$. The $Z / X$ uncertainties are typically somewhat more than a factor of three larger than the optimistic uncertainties.

The uncertainty for the ${ }^{8} \mathrm{~B}$ neutrino flux provides the most dramatic and also the most important example of the differences between the various ways of calculating the flux uncertainties. The traditional $Z / X$ method gives a $20 \%$ flux uncertainty for ${ }^{8} \mathrm{~B}$ neutrinos. Our conservative estimate using individual element abundances is $11.6 \%$. The optimistic estimate for the ${ }^{8} \mathrm{~B}$ composition uncertainty is only $5 \%$ using Asplund et al. (2005) abundance uncertainties. Thus there is a factor of four difference in the size of the estimated flux uncertainty depending upon which method is used to estimate the composition uncertainty.

The uncertainty for the important ${ }^{7} \mathrm{Be}$ neutrino flux also varies by a factor of four depending upon the method of calculation: $8 \%$ ( $Z / X$ estimate), $5.3 \%$ (conservative individual abundance uncertainties), and $2.2 \%$ (optimistic individual abundance uncertainties). 


\subsubsection{The role of correlations}

Correlations among the uncertainties of the different element abundances can affect the calculated uncertainties in the solar neutrino fluxes. The correlations can be taken into account using equation (8), equation (10), and equation (11). We have made crude estimates of the correlations in the present paper. We have used $\rho(k, l)=1$, when $k$ and $l$ are two different elements from among $\mathrm{O}, \mathrm{Ne}$, and Ar. We have neglected all other correlations. With these estimates, correlations are not numerically important in determining the final uncertainties in the solar neutrino fluxes, especially since we advocate using the large, conservative abundance uncertainties given in the second column of Table 4 .

It would be useful in future compilations of element abundances to specify explicitly the correlations between different element abundances and their uncertainties. When the present conflict between recent abundance determinations and helioseismology measurements is resolved and we can adopt more optimistic estimates for the abundance uncertainties, it will be desirable to evaluate accurately, using new data, the effects of correlations on the calculated neutrino fluxes.

\subsubsection{Total neutrino flux uncertainties from all known sources}

Table 8 gives the total uncertainties from all known sources for each solar neutrino flux and for the predicted rate of each radiochemical experiment. The uncertainties for everything except the surface chemical composition of the Sun are taken from Bahcall \& Pinsonneault (2004).

\section{${ }^{8} \mathrm{~B}$ neutrino flux uncertainty}

The uncertainty for the crucial ${ }^{8} \mathrm{~B}$ neutrino flux is most affected by the detailed estimate using individual abundances. In the traditional $Z / X$ method, the total uncertainty in the calculated ${ }^{8} \mathrm{~B}$ neutrino flux is a whopping $23.0 \%$ (actually $24.8 \%$ if we use BP04 partial derivatives rather than the Bahcall \& Ulrich 1988 partial derivatives). Our preferred conservative uncertainty estimate is $16.3 \%$; our optimistic error estimate is only $12.5 \%$.

With our preferred conservative estimate, the composition uncertainty still remains the largest contributor to the uncertainties in the calculation of the ${ }^{8} \mathrm{~B}$ neutrino flux (see Table 2 of Bahcall \& Pinsonneault 2004 for estimates of neutrino flux uncertainties due to all sources). The conservative composition error for the ${ }^{8} \mathrm{~B}$ neutrino flux is $11.6 \%$ (see Table 7 of this paper), which is much larger than the next largest contribution to the uncertainty, 
$7.5 \%$, from the low energy cross section factor for the ${ }^{3} \mathrm{He}(\alpha, \gamma){ }^{7} \mathrm{Be}$ nuclear fusion reaction (see Table 2 of Bahcall \& Pinsonneault 2004).

In order to reduce the composition uncertainty to a level where it is no longer the largest contributor to the ${ }^{8} \mathrm{~B}$ neutrino flux uncertainty, we would need to have confidence in the optimistic abundance uncertainties. For the optimistic uncertainties, the composition error for the ${ }^{8} \mathrm{~B}$ neutrino flux is only $5 \%$ (Table 7 ). But, it is difficult to be confident in the optimistic abundance uncertainties since, as described in the introduction of this paper, the new abundance analyses lead to solar models in conflict with helioseismological measurements.

The reduced estimated uncertainty for the ${ }^{8} \mathrm{~B}$ solar neutrino flux has implications for solar neutrino research since the ${ }^{8} \mathrm{~B}$ neutrino flux is measured by the SNO and SuperKamiokande solar neutrino experiments.

The total ${ }^{8} \mathrm{~B}$ neutrino flux measured by the neutral current mode of the SNO experiment (Ahmed et al. 2004) is

$$
\phi\left({ }^{8} \mathrm{~B}, \mathrm{SNO}\right)=0.90 \phi\left({ }^{8} \mathrm{~B}, \mathrm{BP} 04 \text { solar model }\right)[1.0 \pm 0.09 \pm 0.16]
$$

where the first uncertainty listed in equation (13) is the $1 \sigma$ measurement error and the second (larger) uncertainty is the estimated $1 \sigma$ uncertainty in the solar model calculation (taken from Table 8). If all the data from solar neutrino and reactor experiments are combined together, the above relation becomes (Bahcall et al. 2004a):

$$
\phi\left({ }^{8} \mathrm{~B}, \mathrm{SNO}\right)=0.87 \phi\left({ }^{8} \mathrm{~B}, \mathrm{BP} 04 \text { solar model }\right)[1.0 \pm 0.05 \pm 0.16]
$$

The calculated ${ }^{8} \mathrm{~B}$ neutrino flux (Bahcall \& Pinsonneault 2004) agrees with the measured flux to better than $1 \sigma$. The theoretical uncertainty is much larger than the uncertainty in the measurements.

\section{${ }^{7}$ Be neutrino flux uncertainty}

For the ${ }^{7}$ Be solar neutrinos, which will be measured by the BOREXINO solar neutrino experiment (see Alimonti et al. 2002), the situation is somewhat different. The largest contribution to the presently estimated uncertainty in the predicted flux is $8.0 \%$ from the laboratory measurement of the rate of the ${ }^{3} \mathrm{He}(\alpha, \gamma){ }^{7} \mathrm{Be}$ nuclear fusion reaction (see Adelberger, et al. 1998). Even our conservative composition uncertainty is only $5.3 \%$ for the ${ }^{7} \mathrm{Be}$ neutrino flux (Table 7). However, the situation may change in the near future. A recent measurement of the ${ }^{3} \mathrm{He}(\alpha, \gamma){ }^{7}$ Be reaction by Singh et al. (2005) reports a precision for this 
reaction rate which is much better than 5\%. If the Singh et al. (2005) result is confirmed by future measurements, then even for the ${ }^{7}$ Be solar neutrinos the solar composition will be the largest contributor to the calculational uncertainty.

$$
\mathrm{p}-\mathrm{p} \text {, pep, }{ }^{13} \mathrm{~N} \text {, and }{ }^{15} \mathrm{O} \text { neutrino flux uncertainties }
$$

The conservative composition uncertainty is the largest contributor to the estimated uncertainty in the calculation of the p-p, pep, ${ }^{13} \mathrm{~N}$, and ${ }^{15} \mathrm{O}$ solar neutrino fluxes (compare Table 7 of this paper with Table 2 of Bahcall \& Pinsonneault 2004). However, the optimistic composition uncertainties are less than other uncertainties in calculating these neutrino fluxes.

The p-p solar neutrino flux has been measured by combining the results from all the relevant solar neutrino and reactor experiments, together with the imposition of the luminosity constraint (Bahcall 2002). The result is (Bahcall et al. 2004a)

$$
\phi(\mathrm{p}-\mathrm{p}, \text { all neutrino experiments })=1.01 \phi(\mathrm{p}-\mathrm{p}, \mathrm{BP} 04 \text { solar model })[1.0 \pm 0.02 \pm 0.01]
$$

where the first uncertainty listed in equation (15) is the $1 \sigma$ measurement error and the second (smaller) uncertainty is the estimated $1 \sigma$ uncertainty in the solar model calculation (taken from Table 8).

\section{Comparisons of Total Uncertainties from Different Methods}

Table 8 shows that for all the solar neutrino fluxes produced in the p-p chain except the ${ }^{8} \mathrm{~B}$ neutrino flux (i.e., the p-p, pep, hep, and ${ }^{7}$ Be neutrino fluxes), the total uncertainties from all sources are similar for the conservative and the $Z / X$ estimates for the composition uncertainties. Of course, this must be the case if the composition uncertainties are only a small contribution to the total uncertainties. Indeed for hep neutrinos, the calculated rate of the fusion reaction is the dominant recognized uncertainty, 15.1\% (see Bahcall and Pinsonneault 2004; Park et al. 2003). But, as we have seen above, the composition uncertainties are at present the largest contributor to the total uncertainties for all neutrino fluxes except the hep and the ${ }^{7} \mathrm{Be}$ neutrinos.

The reason that the conservative and the $Z / X$ methods give such similar results is that the recent change in the abundances of the volatile elements affects in a similar way the historical average of $Z / X$ and the difference between previous standard and current element abundances. The recently inferred changes in the volatile element abundances represent the largest modern revision in the solar element abundances from one epoch of abundance determinations to its successor. 


\subsection{Do neutrino measurements tell us which abundances are correct?}

The values of the solar neutrino fluxes predicted by solar models depend upon the assumed heavy element abundances. Unfortunately, the agreement between solar neutrino predictions and solar neutrino measurements is excellent for calculations made with both the Grevesse and Sauval (1998) abundances and with the Asplund et al (2005) abundances.

In both cases, the differences between measurements and predictions are smaller than the uncertainties in the predictions and measurements (Bahcall \& Pinsonneault 2004; Bahcall, \& Basu Serenelli 2005).

In fact, Table 1 of Bahcall \& Pinsonneault (2004) gives the neutrino fluxes for a state-ofthe art solar model that was computed using the older Grevesse \& Sauval (1998) abundances (BP04) and the more recent Asplund et al. (2005) abundances. If we compare the fluxes from these two models, we see that in all cases their neutrino fluxes agree to within $1 \sigma$ of the total theoretical uncertainties given in the first column of Table 8.

\subsection{Final word}

We conclude, as we began, by emphasizing that composition uncertainties are the most important uncertainties for determining the accuracy of solar neutrino calculations. It would be desirable if multiple groups would undertake determinations of solar abundances in order that systematic uncertainties can be more readily assessed. The possibility of obtaining improved solar spectroscopic data should also be considered. In the future, the correlations between the uncertainties in different element abundances should be specified explicitly by researchers determining the abundances. The correlations, which hopefully will become relevant for precise evaluations of reduced neutrino flux uncertainties, can be taken into account using the formulae given in this paper.

J. N. B. and A. M. S. are supported in part by NSF grant PHY-0070928. We are grateful to B. Draine, N. Grevesse, K. Lodders, C. Pena-Garay, M. Pinsonneault, and D. Sasselov for valuable discussions. We are grateful to E. Lisi and M. Pinsonneault for insightful comments on a draft of the manuscript.

\section{REFERENCES}


Abraham, Z., \& Iben, I., Jr. 1971, ApJ, 170, 157

Adelberger, E. G., et al. 1998, Rev. Mod. Phys., 70, 1265

Ahmed, S. N., et al. (SNO Collaboration) 2004, Phys. Rev. Lett., 92, 181301

Alimonti, G., et al. 2002, Astropart. Phys., 16, 205

Allende Prieto, C., Lambert, D. L., \& Asplund, M. 2001, ApJ, 556, L63

Allende Prieto, C., Lambert, D. L., \& Asplund, M. 2002, ApJ, 573, L137

Asplund, M. 2000, A\&A, 359, 755

Asplund, M., Grevesse, N., \& Sauval, A. J. 2005, in Cosmic Abundances as Records of Stellar Evolution and Nucleosynthesis, ASP Conference Series, eds. F. N. Bash, \& T. G. Barnes, astro-ph/0410214

Asplund, M., Grevesse, N., Sauval, A. J., Allende Prieto, C., \& Kiselman, D. 2004, A\&A, 417,751

Asplund, M., Nordlund, A., Trampedach, R., \& Stein, R. F. 2000, A\&A, 359, 743

Badnell, N. R. et al. 2004, astro-ph/0410744

Bahcall, J. N. 1964, Phys. Rev. Lett., 12, 300

Bahcall, J. N. 1966, Phys. Rev. Lett., 17, 398

Bahcall, J. N. 1989, Neutrino Astrophysics (Cambridge, UK: Cambridge University Press)

Bahcall, J. N. 2002, Phys. Rev. C., 65, 025801

Bahcall, J. N., Bahcall, N. A., \& Shaviv, G. 1968, Phys. Rev. Lett., 20, 1209

Bahcall, J. N., Basu, S., Pinsonneault, M. H., \& Serenelli 2005, ApJ, in press, astro$\mathrm{ph} / 0407060$

Bahcall, J. N., Cooper, M., \& Demarque, P. 1967, ApJ, 150, 723

Bahcall, J. N., Gonzalez-Garcia, M. D., \& Peña-Garay, C. 2004a, J. High Energy Phys., 08, 016

Bahcall, J. N., Huebner, W. F., Lubow, S. H., Parker, P. D., \& Ulrich, R. K. 1982, Rev. Mod. Phys., 54, 767 
Bahcall, J. N., \& Pinsonneault, M. H. 1992, Rev Mod Phys, 64, 885

Bahcall, J. N., \& Pinsonneault, M. H. 1995, Rev Mod Phys, 67, 781

Bahcall, J. N., \& Pinsonneault, M. H. 2004, Phys. Rev. Lett., 92, 121301

Bahcall, J. N., Pinsonneault, M. H., \& Basu, S. 2001, ApJ, 555, 990

Bahcall, J. N., Serenelli, A. M., \& Pinsonneault, M. H. 2004b, ApJ, 614, 464

Bahcall, J. N., \& Serenelli, A.M. 2005, ApJ Letters (in press), astro-ph/0412440

Bahcall, J. N., \& Ulrich, R. K. 1971, ApJ, 170, 593

Bahcall, J. N., \& Ulrich, R. K. 1988, Rev. Mod. Phys., 60, 297

Basu, S., \& Antia, H. M. 2004, ApJ, 606, L85

Boothroyd, A. I., \& Sackmann, I. J. 2003, ApJ, 583, 1004.

Couvidat, S., Turck-Chieze, S., \& Kosovichev, A. G. 2003, ApJ, 599, 1434

Fiorentini, G., \& Ricci, B. 2002, Phys. Lett. B, 526, 186

Fukuda, S., et al. 2001, Phys. Rev. Lett., 86, 5651

Grevesse, N., \& Sauval, A. J. 1998, Space Sci. Rev., 85, 161

Lodders, K. 2003, ApJ, 591, 1220

Park, T. S., et al. 2003, Phys. Rev. C, 67, 055206

Schlattl, H., \& Weiss, A. 1999, A\&A, 341, 272

Sears, R. L. 1964, ApJ, 140, 477

Seaton, M. J., \& Badnell, N. R. 2004, MNRAS, 354, 457, astro-ph/0404437

Singh, B. S. N., Hass, M., Nir-El, Y., \& Haquin, G. 2005, nucl-ex/0407017

Thoul,A. A., Bahcall, J. N., and Loeb, A. 1994, ApJ 421, 828

Turcotte, S., \& Christensen-Dalsgaard, J. 1998, in Structure and Dynamics of the Interior of the Sun and Sun-like Stars, Proc. SOHO\&GONG Workshop, ed. S. Korzennik, \& A. Wilson [ESA SP-418, Noordwijk: ESA] 561

Watanabe, S., \& Shibahashi, H. 2001, Publ. Astron. Soc. Japan, 53, 565 
Young, P. A., \& Arnett, D. 2005, ApJ (in press), astro-ph/0409658

This preprint was prepared with the AAS IATEX macros v5.2. 